\title{
THE Simulation-baSed SMART MANAGEMENT APPROACH for Cellular Network Operation and PlanNing
}

\author{
Alexander Suleykin \& Peter Panfilov
}

Higher School of Economics, Myasnitskaya Ulitsa, 20, Moscow, 101000, Russia


This Publication has to be referred as: Suleykin, A[lexander] \& Panfilov, P[eter] (2017). The Simulation-Based Smart Management Approach for Cellular Network Operation and Planning, Proceedings of the 28th DAAAM International Symposium, pp.0423-0432, B. Katalinic (Ed.), Published by DAAAM International, ISBN 978-3-902734-11-2, ISSN 1726-9679, Vienna, Austria

DOI: $10.2507 / 28$ th.daaam.proceedings.059

\begin{abstract}
The number of mobile users in the world has been steadily increasing for last decades and is anticipated to continue its growth by the end to 2019 up to more than 5 billion users. This process brings new challenges to the telecom companies over the globe and to the cellular network infrastructures. The problem (dead) zones and dropped calls are now among the most critical issues that mobile providers tend to solve, while trying to increase the quality of service at the same time. The paper discusses modeling approaches to the cellular network's problem zones detection and analysis, on the basis of complaints, subscribers and base stations data aggregation and analysis. We describe algorithms to be used in building Heat Map geo-spatial visualizations of cellular network data, which show the distribution of problem zones within the network with their corresponding weights assigned according to the amount of complaints and population density in the area. This information is used in prioritization of distributed problem zones for the cellular network development and decision support on new base station deployment. It means that simulation-based approaches to problem zone detection and fixation in cellular network can help to increase the customer satisfaction of connection level and quality of mobile service. In addition, the paper presents the evaluation of economic effect of solving the problem zone issue that follows the subscriber traffic increase.
\end{abstract}

Keywords: cellular network; base station; problem zones; visual simulation; smart management

\section{Introduction}

In recent years, the popularity of mobile phones is dramatically increasing together with new technology development such as Internet of Things and Machine-to-Machine communications. The number of smartphone users has already reached 4.61 billion users in 2016, and upward trend is forecasted for the market with 5.07 billion users by the end of 2019 [1]. This trend might be explained by the new technologies appearance on the market with higher data speed transmission (for example 4G, 4G-Advanced, 5G creation etc.), the decrease of smartphone prices, the decrease of connection prices and others. As a result, smartphones are becoming more and more achievable for people with different 
income, and more users are appearing on the market. However, this process generates some new challenges for mobile operators.

As amount of smartphone and mobile users is growing and forecasted to continue growing at least until 2019, the load on the wireless mobile network is also increasing. This is a huge challenge that mobile operators are eventually facing and try to troubleshoot and overcome.

There is an obvious need for each mobile operator to provide the maximum coverage of the entire territory of the operator's presence with mobile network services. However, there is an issue of the planning optimal allocation of new base stations in problem zones of the network that needs to be done taking into account the following factors:

- The budget that is to be invested in construction of new stations. The construction of base stations is expensive and for mobile operators it is highly important to place new base stations in locations that cover problem zones and provide maximum user satisfaction at minimum cost.

- The minimization of the churn's average revenue per user (ARPU) value, which is supposed to decrease in cases when users decide to leave mobile operators for the bad connection in some locations. If user decides to alter the mobile provider, the ARPU of this user should be minimal. Thus, there is a case to find users with different ARPU values, prioritize them and try to satisfy the quality of connection for the most critical users for company from this list - the users with the highest ARPU.

- The decrease of number of such unsatisfied users who can face the dead connection problem.

- The current distribution of base stations.

- Subscriber's complaints registered on the web-site of the company, Contact Center or Speedtests (special bespoke company's measurements). In this paper, only these primary sources for complaints are considered. There are also many tools existing on the market, which allow to analyze the network quality in a more automated way (using special apps), but it will not be in scope of this study.

So the optimal planning of new base stations construction and deployment is extremely important for the telecom company, bringing better customer satisfaction, decreasing the churn losses and more effectively distributing connection power within the network.

Usually, telecom companies have their own private methods for problem zones detection and prioritization and not too much work has been done so far that is open for public. A lot of work were devoted to the femtocells usage [2, 3, 4] for dead zones coverage, mobile monitoring [5], some network delays caused by different factors [6], network optimization near wireless dead zones [7], architecture and its management in mobile networks [8, 9, 10].

One of the main challenges of operating a cellular network $(\mathrm{CN})$ is associated with the fluctuations of user demand and the signal strength from the base station (BS), which may significantly depend on the environmental conditions. These issues make the management of the $\mathrm{CN}$ in terms of the operation and planning difficult. With this regard, a management strategy for $\mathrm{CN}$ operation and development that can mitigate these fluctuations and guarantee the $\mathrm{CN}$ operation in the most cost-effective manner is a necessary. In this research, a novel simulation-based data-driven smart management (DDSM) approach is proposed for determining problem zones in a CN, their prioritization for further use in planning new BS construction and deployment.

Our proposed approach is equipped with a database, a simulation model with visual simulation module, and an optimization module. In the database, data related to the CN's topology (i.e., subscriber demand and complaint, mobile traffic parameters, etc.), as well as those obtained from the measurements (i.e., signal strength from BS, environmental sensors, etc.) are stored. In the simulation, the operation of the considered $\mathrm{CN}$ is simulated under various system uncertainties and problem zones are detected and visualized. Meanwhile, the complexity of the optimization problem is determined and the optimization module is executed to obtain the optimal operation plan for the CN and plan for new BS construction and deployment in terms of the minimum operating/construction cost. In order to solve the optimization problem, a problem zone prioritization-based multi-objective optimization algorithm for operation planning of the CN is proposed. The results of the optimization module are finally sent back to the $\mathrm{CN}$ system for execution. The performance of the proposed approach is demonstrated via a synthetic CN. The approach has been constructed in a generic manner so that it can be employed by any $\mathrm{CN}$ system that has similar types of base stations by importing the necessary data capturing its characteristics (i.e. number of BSs installed), topology, and environmental conditions into the database.

The remainder of this paper is organized as follows. First we define the problem zones within the CN domain as those areas where quality of service parameters in mobile networks can be compromised due to bad subscriber's connection to base stations. We then discuss a related and important area - the protection of CNs from losing subscribers, followed by an idea of data-driven simulation and data visualization integration in a management process of the $\mathrm{CN}$ operation and planning to develop and model a reliable $\mathrm{CN}$. Then we present our visual simulation-based approach to the problem zone detection and prioritization in the $\mathrm{CN}$ using examples of real-world geographical areas. Finally, we discuss methodological issues of the DDSM approach, especially in the context of economic impact of smart management of the $\mathrm{CN}$. We will talk about the $\mathrm{CN}$, its features and how problems in future can be overcome.

The architecture of proposed approach can be described as follows (Figure 1): 


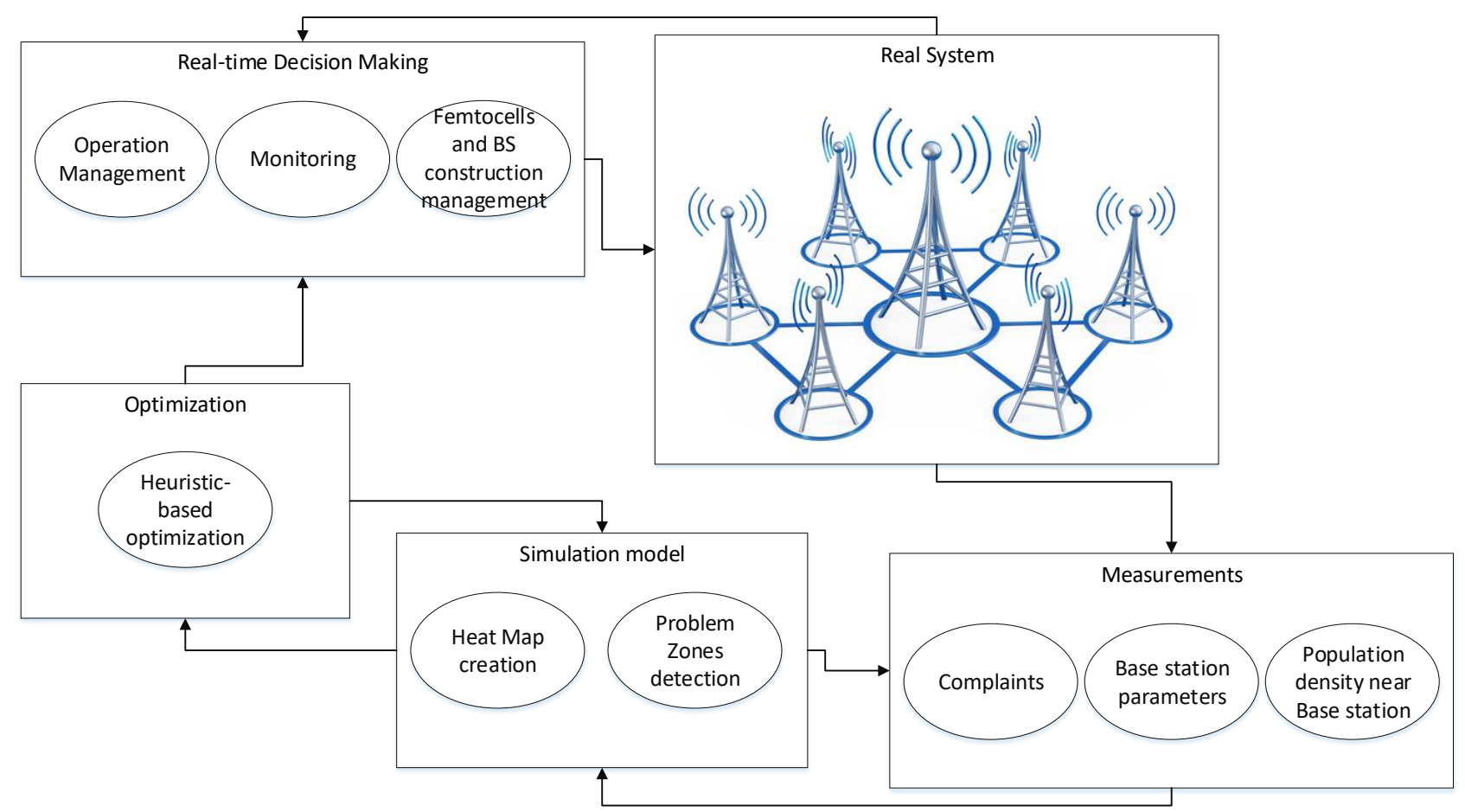

Fig. 1. Overview of DDSM approach for cellular network operation and planning

\section{The domain peculiarities and problem description}

\subsection{Dead Zones}

Basically, locations where mobile phones or User Equipment cannot transfer signal to the near base station or repeater are named dead zones. In these particular locations, the mobile connection is severely restricted or not available and there is no possibility for user to transmit data or have a voice call. Mostly it is caused by the fact that the signal in these zones is blocked by some buildings, hilly terrains or other obstacles that interfere with the correct signal transmission from User Equipment to base station.

There are many factors that can influence dead zones. They can be detected even in the locations, where a wireless carrier offers coverage with existing network technology and topology. Thus, it might be caused by the interference of other mobile sites, limited density of base stations in mobile network, or even the locations and capacity of antennas hanging on base station with particular height.

As a matter of fact, the transmission of data in cellular network is based on radio waves transmission, which can be dramatically reduced with distance and it makes mobile network transmission unreliable at times. Hilly terrains, high trees and buildings, even wind and seasonal changes can have an influence on data transmission and interfere, especially in urban areas, with its high density, clearly disturbing the unhindered and smooth radio waves distribution.

All these factors force mobile providers to continually work on the improvement of their network, upgrading existing ones and constructing new mobile sites. The purpose of this work is to reduce the amount of dropped calls, dead zones (or problem zones or coverage holes) and prevent from failures on the cellular network.

\subsection{Dropped calls}

Dropped calls are the calls that were terminated and caused by network issues, usually associated with "Dead Zones". It is highly important issue for mobile operators nowadays, even though the amount of such drops has been significantly reduced for last 20 years. This termination is considered as unexpected and technically calls "Abnormal release".

There are many reasons for dropped calls including the following:

- Mobile subscriber is located outside the coverage area after setting up a connection - this is simply the area, where radio towers can not receive the signal because of the distance. The standard base station in ideal conditions can provide a coverage radius of up to $35 \mathrm{~km}$, and with additional software options - up to $120 \mathrm{~km}$, which is in real cases rarely achievable because of interferences. The coverage radius of the micro-cell does not exceed 1 kilometer with good antenna.

- Drops during handovers between cells within one technology $(2 \mathrm{G} / 3 \mathrm{G} / 4 \mathrm{G})$ or drops during handovers from one technology to another. 
- The lack of capacity of the network in some particular areas. Base stations and cells might be overloaded with traffic and amount of subscribers they serve, and in this cases become unavailable for other subscribers in this zone. Incorrect configuration or huge number of users being served by this base station might cause this issue.

- Transmission problems that might be caused by weather conditions or terrain structure. This issue is related to the radio waves interferences between User Equipment and base station.

- Loss of battery power at one of the ends of the call.

Dropped calls are considered by mobile providers as one of the most critical complaints from the user point of view. Many subscribers, which are experiencing dead zones or dropped calls very often tend to leave this particular mobile operator. Therefore, such companies are exceedingly interested in solving issues with dropped calls and dead zones. However, it is not possible to entirely solve all issues but the aim is to reduce amount of dropped calls and dead zones.

\section{Proposed Approach}

To address issues of problem zone detection and fixation in cellular network we propose a data-driven smart management (DDSM) approach which is based on modeling and analyzing particular business process of telecom company with a specific focus on the complaints management and planning new base station constructions and deployments. This business process is more or less similar in most of telecom companies (with regard to the user complaint processing and some network Speedtests), so the following workflow model of business process is applicable to any telecom company that acts as telecom operator and analyzes user's complaints and network performance:

1. At the end of each quarter the Department of Quality and Clients Experience (DOQCE) uploads the necessary complaints / measurements for the required period, collects them in one file in a certain format (CSV-format). The DOQCE downloads file with complaints from the Relational database, where all data from Speedtests and different complaints collected from the Contact Center and web-site are aggregated.

2. The DOQCE loads file for data processing, which is executed using simulation-based data analytics system.

3. The simulation based data analytics system performs the problem zone detection and analysis using data from existing and projected BSs. The reports on simulation results are generated for decisions on new BS deployment.

4. Based on reports and data visualizations received as an output of the simulation system, the Technical department at telecom company decides about where exactly it is critically to construct new base station or microcell so to increase the quality of service in the network.

5. After the construction of new base station or microcell, the effect of this station installation is evaluated.

In particular, the proposed DDSM approach is equipped with three modules: 1) a database including the CN's topology data (i.e., subscriber demand and complaints, mobile traffic parameters in the area, etc.), network measurements (i.e., Speedtest data), and (optionally) the data for environmental factors (i.e., solar radiation, wind speed, temperature, etc.); 2) a simulation system with data visualization modul, in which operation of the $\mathrm{CN}$ is simulated and problem zones are detected and visualized; and 3) a multi-objective optimization module which finds the near-optimal operation plan and new BS deployment plan in terms of minimum cost using a problem zone prioritization-based algorithm. The results obtained from the optimization module are sent back to the $\mathrm{CN}$ system to enhance its operation. The experiments conducted in this study demonstrate the power of the proposed approach in real-time assessment and control of operation and development management in CNs. The components of our proposed approach are presented in detail in this section.

\subsection{Data Base}

For easy adaptation of the proposed approach to varying types of CNs with different characteristics, the heterogeneous data associated with the considered CN's topology, operations, and environment are collected and stored in a database. The database contains the $\mathrm{CN}$ topology data (i.e., subscriber demand and complaint, mobile traffic parameters, BS locations and power, Speetest data, etc.) and the operational data for the BSs that characterize the load demand and operating cost of various stations. The environmental data (i.e., wind speed, solar irradiance, temperature, etc.) can also be collected and stored to estimate of the strength of signal that can be received from the base stations. For the data, both data from synthetic functions (i.e., probability distribution functions, pdf) and a real-world measurement data recorded from the sensors in specific geo-locations or obtained from special apps (e.g., Speedtest) can be used.

It is assumed that all collected data is stored in a single database in unified format in order to prevent any data manipulations before uploading it in a simulation.

There are three major sources of data in a database: subscribers (demand and complaints), base stations and mobile traffic in the area, and (optionally) environment.

The regarded primary sources for user complaints as have been listed above were data from company Contact Centers, web-site of the company and Speedtests data. Subscribers' data sectioned by stationary and stirring as well as data on the base stations for analyzed region is being received from relational database of a telecom company. The following base 
stations attributes are needed for a model: cell id, cell lac, standard network, base station name, launch date, latitude, longitude, area. The number of subscribers and the density of complaints are sectioned by squares of 500 x 500 meters (polygons). The proposed formula is as following:

$$
\text { Weight }=(1-(X * \text { Subs_Stay }+Y * \text { Subs_Move }) / 2 * \text { Zone_count_claims }),
$$

\section{Where}

Weight - the average proportion of complaints per subscriber,

$X$ - the proportion of stationary subscribers,

Subs_Stay - the amount of stationary subscribers,

$Y$ - the proportion of stirred subscribers,

Subs_Move - the amount of stirred subscribers,

Zone_count_claims - the amount of complaints in this area.

The Weight metric shows the density of complaints per squares (tiles) of 500 x 500 meters, which can be easily visualized using Python. For the square of 500 x 500 meters, there is a distribution of complaints over time. The number of complaints with a stable problem is distributed according to Poisson's law and about $5 \%$ of complaints come out. In days, when the level of complaints exceeds $99 \%$ of the confidence interval, the number of complaints is reduced to an average of one quarter.

Radius to the nearest BS is a minimal distance to the nearest base station. The sense in determining the size of the problem zone is the following: the father is a zone from the base station - the wider and broader the problem area.

\subsection{Simulation model for the problem zone detection in the $C N$}

A simulation model provides an accurate representation of the considered $\mathrm{CN}$ (as a whole or a specific part of it) while simultaneously capturing the behavior of operating components as well as their interactions with each other. In this study, the operating components including demand (subscribers) and service providers (base stations) are designed as simulation models (e.g., the software agents in case of agent based modeling, ABM).

In the core of the simulation system, there is a data visualization system that provides interactive geo-spatial display of so called problem zones detected and identified as a result of real-time measurements and simulation.

Heat Map visualization is a Geo-spatial visualization of a map with imposing weight values as different colors per every square on this map.

The selected data visualization and analysis tool is Python with its powerful libraries for data processing and handling. The advantage of generating Heat Maps in Python is that there is the ability to fine-tune parameters (any nucleus, and not only monotonous), generating squares and polygons of any shape.

Hive is used as SQL-like database on top of Hadoop. But it is also possible to store polygons in any relational database.

GeoSpark is an open source in-memory cluster computing system for processing very large-scale spatial data. A fundamental data structure of Apache Spark is the Resilient Distributed Datasets (RDD) an immutable distributed collection of objects. Each dataset in RDD is divided into logical partitions, which may be computed on different nodes of the cluster. GeoSpark extends RDDs to form Spatial RDDs (SRDDs) and efficiently partitions SRDD data elements across machines and introduces novel parallelized spatial transformations and actions (for SRDD) that provide a more intuitive interface for users to write spatial data analytics programs [11].

The final visualization is performed in QGIS. QGIS is a professional GIS application that is built on top of and proud to be itself Free and Open Source Software (FOSS). QGIS is a user friendly Open Source Geographic Information System (GIS) licensed under the GNU General Public License. QGIS is an official project of the Open Source Geospatial Foundation (OSGeo). It runs on Linux, Unix, Mac OSX, Windows and Android and supports numerous vector, raster, and database formats and functionalities [12].

Table 1 shows the steps of the generalized procedure of visual simulation modeling of cellular network performance using network data collected and prepared as it was explained earlier.

The first step in visual simulation of a problem zones in cellular network is a Heat Map visual model generation in python with a bi-weight core with a radius and weight values taken from the attributes of the point of complaints.

Then separate tiles of a Heat Map are combined into zones and certain metrics are counted. According to the distance between the centers of tiles, tiles are united when having one common side. Upon completion of the union operation on tiles, a new zones are formed, the maximum density of the Heat Map is calculated which is exceeding the average level of complaints.

Next step involves splitting large zones into smaller ones and counting convex shapes, which is related to the model optimization strategy.

After model is optimized, special shape files are generated as an output from python and GeoSpark tools. These shapes files presents the layers in QGIS - BSs, complaints and Heat Maps. In QGIS this layers are imposed on the geographical map (physical, street, hybrid or satellite) and problem zones might be easily analyzed. 


\begin{tabular}{|c|c|c|}
\hline Step & Technology & Parameters \\
\hline Convex shape of segments & GeoSpark & \\
\hline Dynamics of complaints in zone & GeoSpark & \\
\hline $\begin{array}{l}\text { Combining the pieces (tiles) of the heat map } \\
\text { into zones }\end{array}$ & GeoSpark & \\
\hline $\begin{array}{l}\text { Cutting the top of the heat map (filtering the } \\
\text { tiles) }\end{array}$ & GeoSpark & cut-off percentage \\
\hline Counting the final metric & GeoSpark & $\begin{array}{l}\text { cell size of heat map kernel function for } \\
\text { blurring a complaint blur radius }\end{array}$ \\
\hline $\begin{array}{l}\text { Building heat maps } \\
\text { (generation of tiles) }\end{array}$ & GeoSpark & $\begin{array}{l}\text { weight-of-complaint function method to } \\
\text { find the nearest base station }\end{array}$ \\
\hline Complaint attributes calculation & GeoSpark & upper area boundary \\
\hline Segmentation of large zones into chunks & Python & \\
\hline Removing intersections of convex hulls & GeoSpark & \\
\hline $\begin{array}{l}\text { Filtration of complaints on short-term } \\
\text { accidents }\end{array}$ & Python & p-value - affects the proportion of cut-off \\
\hline Storing polygons & Hive tables & number of last calculations for storage \\
\hline Final visualizations & QGIS & \\
\hline
\end{tabular}

Table 1. Visual simulation workflow, used technology and parameters

\subsection{Optimization strategy of the CN upgrade}

In this study, the optimization of the $\mathrm{CN}$ operation and upgrade is executed using data from the identified problem zones in the $\mathrm{CN}$ to determine the new BS's construction plan in terms of the minimum cost of construction and maximum traffic increase in a particular area. These multi-scale problems are solved via heuristic-based optimization algorithm that uses problem zone prioritization technique.

The optimization is basically based on the level of the convex of map shapes. If the convex is more than the threshold, than we consider this zone a problem with particular weight. Using k-averaging technique tiles are divided into groups and convex hulls are counted. If the area of the convex hull is more than 4 square kilometers, the number of clusters in $\mathrm{k}$ means increases by 1 .

In QGIS, the zones obtained from the same broken large zone are checked for intersections. A random one of a pair of zones is replaced by its difference with the other. Thus, the model after optimization shows only convex geo-spatial zones with exceeds of threshold, which are the objects of this research - prioritized problem zones itself.

In future, we plan to explore the possibility of using both traditional and new "big data" approaches (for example, based on machine learning) to optimization problems in DDSM system for managing cellular networks.

\section{Experiments and Results}

\subsection{Experimental setup}

In order to demonstrate the capabilities of the proposed approach, experiments are designed and carried out over a synthetic CN. Each model execution is performed on particular region of a country or city, so the output of the model will show the prioritized problem zones distribution in a selected region.

The selected hardware is GeoSpark an open source in-memory cluster computing system with 30 cores and more than 250 GB RAM. All computations for processing very large-scale spatial data require a lot of RAM, processing cores and storage memory. The selected database is Hadoop with Hive for SQL-data structure as part of pre-installed Hortonworks Big Data application tools. Hortonworks is a leading innovator in the industry, creating, distributing and supporting enterprise-ready open data platforms and modern data applications [13]. So all chosen data for experiments are stored in Hadoop, and all data processing and algorithmic computations are performed on powerful cluster (edge nodes) using Hortonworks applications - Spark, Python.

For the experiments we have selected two geographical regions in the European part of Russian Federation, namely: the southwestern area of Moscow city as an example of huge (over 12 million inhabitants) city with a dense population (over 44 million mobile subscribers/SIM cards), and Stavropol - the medium-sized city (under 500,000 inhabitants) in the southern European region of Russian Federation with relatively low population density (2,7 million inhabitants in the territory of 66,500 sq. kilometers with some 4 million SIM cards). 


\subsection{Modeling results}

The first selected region Stavropol has the following model input parameters (randomly generated numbers):

- Amount of complaints is 19668 (demand agents);

- Amount of cells is 9368 that is related to 1000 base stations (service agents).

The model output is QGIS geo-spatial visualization of problem zones. In our experiment we have received 339 Problem Zones. The output visualizations are the following: the Figure 2 shows the QGIS base stations and complaints distribution on geo-map for Stavropol city:

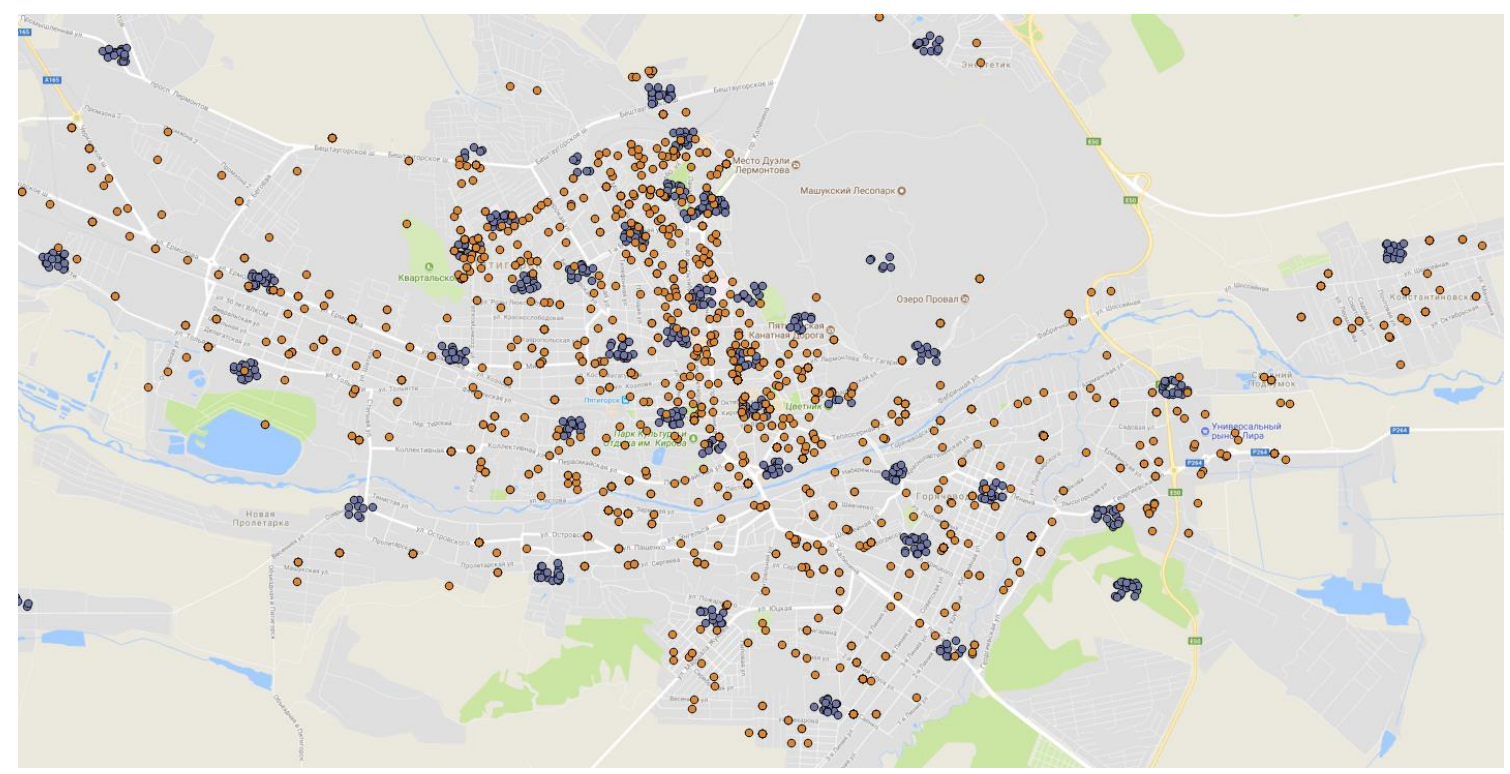

Fig. 2. The QGIS base stations and complaints distribution on geo-map for Stavropol city

The Heat Map visualization itself shows the density distribution of complaints per polygon (500x500 sq. meters):



Fig. 3. The QGIS Heat Map for Stavropol city

And finally, filtering Heat Map by maximum complaints concentration gives the final Problem zones map (fig. 4): 


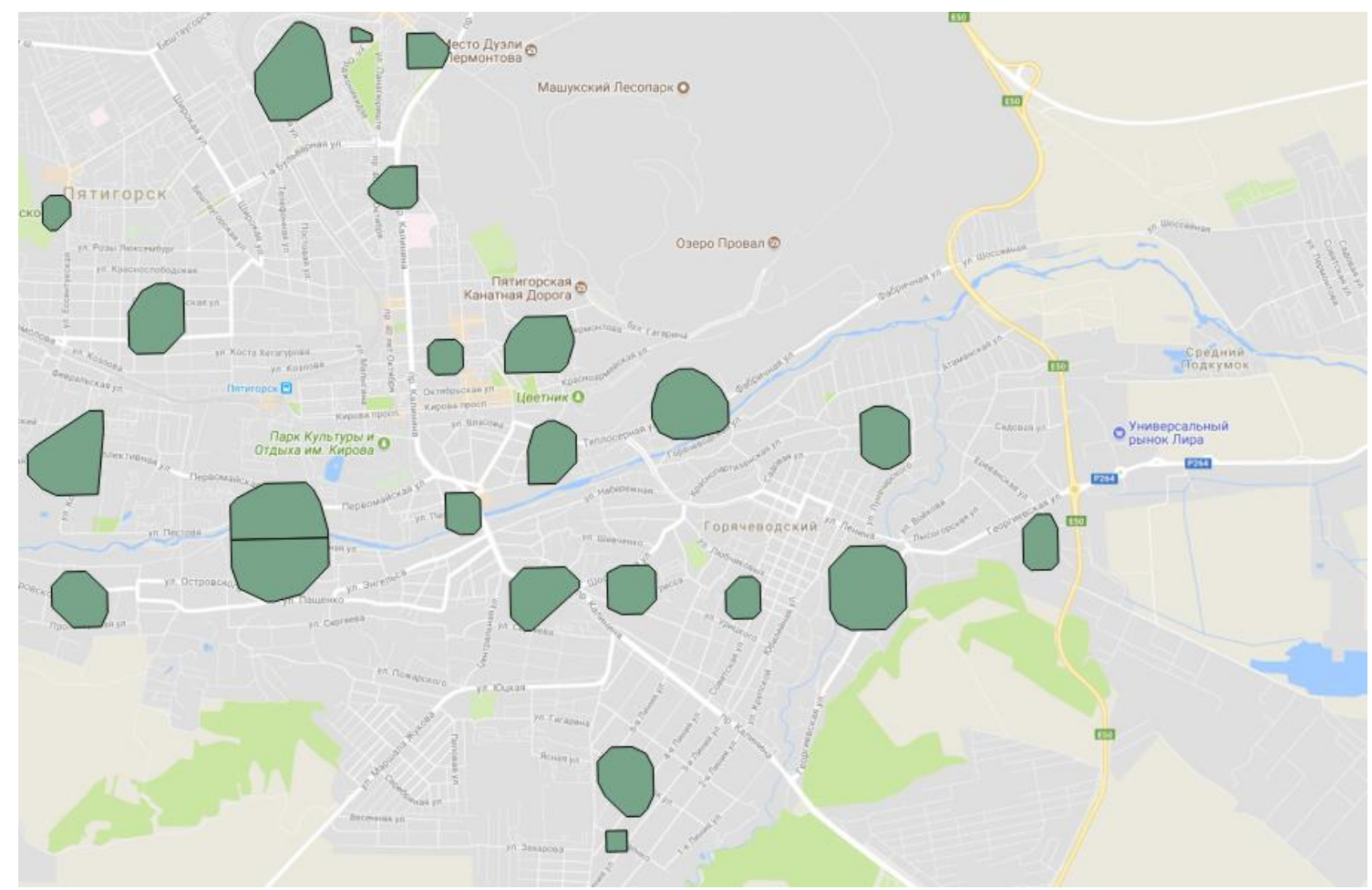

Fig. 4. The QGIS Problem Zones Map for Stavropol city

Additionally, the second experiment was performed for the geographic area in southwestern part of Moscow city. The Fig. 5(a) illustrates the basic final Heat Map visualization of network simulation data, Fig. 5(b) shows visualization of Problem Zones distribution in the area, and Fig. 5(c) depicts the potential effect from problem zone solution.

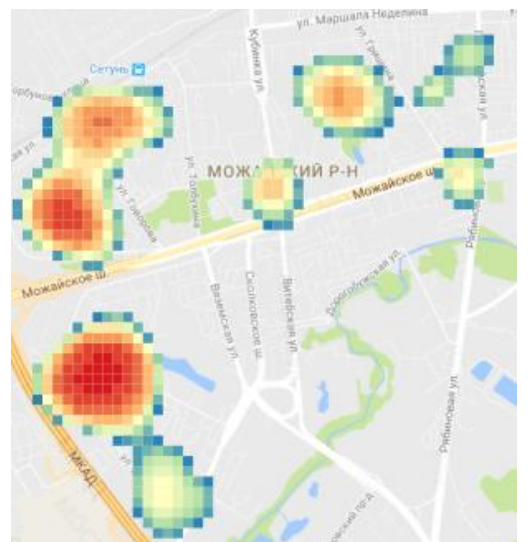

(a)

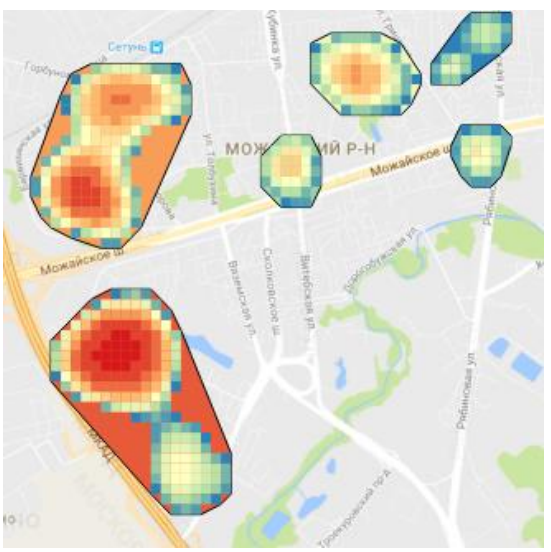

(b)

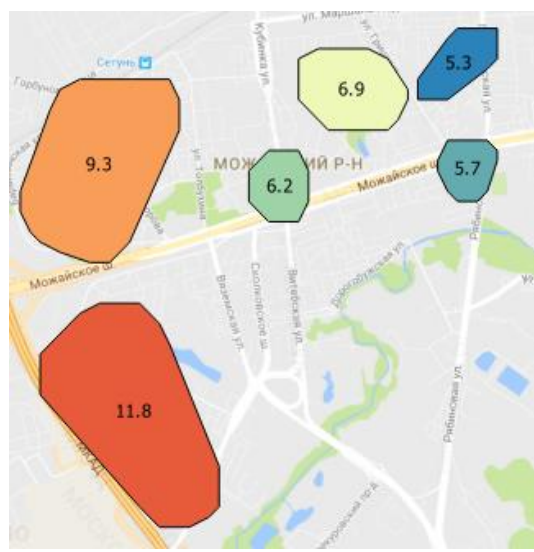

(c)

Fig. 5. The Heat Map visualization of problem zones: (a) the basic Heat Map; (b) Heat Map with problem zone borders; (c) Heat Map with projected complaint decrease numbers.

\section{The approach to the economic effect calculation}

Here we use an "economic effect" parameter as a major criteria for optimization strategy efficiency which is based on the following considerations:

- The problem zones are formed based on geo-analysis data of the feedback received from customers;

- In these zones there is an active unsatisfied demand for communication services;

- If we solve the problem in this zone, we satisfy this demand and the traffic growth in this zone is supposed to be higher than average traffic increase.

This kind of heuristics brings us to the following common sense approach to evaluating and managing economic efficiency of new base station installations in problem zones: 
a. For each solved problem zone issue the base station(s) that serve(s) this zone are determined;

b. For this base station(s), the total data traffic for 6 months is calculated - preceding the issue solving and after 6 months;

c. The growth of traffic "before - after" in the problem zone is compared with the growth of traffic in the region as a whole over the same period;

d. The difference in the rate of growth is counted as an incremental effect from the elimination of the problem zone.

The following picture depicts data traffic dynamics in both problem zone and the average in region:

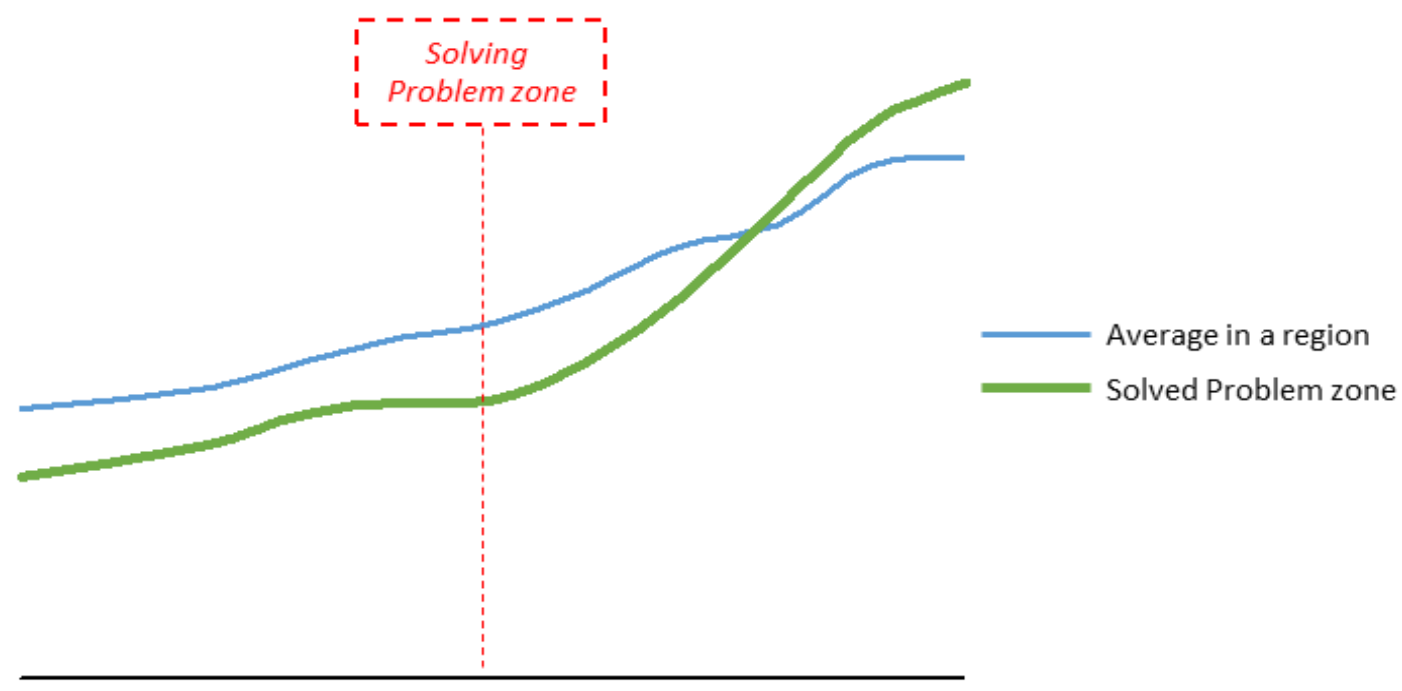

Fig. 6. Data traffic dynamics in both problem zone and the average in a region

\section{Conclusion}

In this work, a DDMS approach is proposed for the problem zone detection and solution within a cellular network. The proposed approach involves a database that stores the system-related operational, topological, and environmental data; a simulation system modeling the operation of the $\mathrm{CN}$ under uncertainties of the signal strength from the BSs and fluctuation of the subscriber demand; and an optimization module that solves the problem of allocation of new BSs in specific area according to the problem zones in the $\mathrm{CN}$ identified by the simulation. The performance of the proposed approach is demonstrated via a synthetic CN with simulated base stations and user demands. Results have shown that the proposed approach has the capability to provide optimal plan for the $\mathrm{CN}$ upgrade without compromising the quality of service requirements of the network.

The DDSM approach is developed in a very generic manner so that it could be adopted and implemented for any CN with similar types of BSs. The presence of one universal model prioritizes problem zones using Heat Maps taking into account the amount of complaints and the population density in particular zone. This model can be applied in any telecom company using proposed models, algorithms, GeoSpark and Python tools. Prioritized problem zones can be used for better distribution of new base stations construction or femtocells, which lead to increase of quality of service, mobile network and customer satisfaction.

In this study, problem zones in the $\mathrm{CN}$ are detected and visualized only on the basis of customer complaints data, but it is also important to estimate the quality of network using more automated solutions, taking into account only network quality parameters related to some particular geolocation. Thus, the automation of complaints collecting, architecture and altering the model accordingly are the further research topics. Supposedly, this will lead to more proper distribution of problem zones on the map and their weights. In the future, the use of new "big data" approaches such as machine learning and deep learning to the optimization problems in DDSM system will be explored. Also the environmental parameters in the $\mathrm{CN}$ will be taken into consideration in simulation when determining problem zones in the $\mathrm{CN}$ and the new $\mathrm{BS}$ construction and deployment plan.

\section{References}

[1] https://www.statista.com/statistics/274774/forecast-of-mobile-phone-users-worldwide (2017). Number of mobile phone users worldwide from 2013 to 2019 (in billions), Accessed on: 2017-10-11.

[2] M. Nagieb, M. Shokair (2014). Improvement of Coverage and Mobility in LTE-A Femto-Cell based on Cognitive Radio Network. International Journal of Computer Applications Vol. 86 No. 11 pp. 37-40 Jan 2014. 
[3] J.M. Benita, V.M.A. Rajam (2017). Road Gateways for femtocell clusters to enable communication and handover in dead zones, Cluster Computing, 2017, ISSN 1386-7857.

[4] J. Benita, V. Rajam (2014). A novel architecture for enhancing communication in dead zones using femtocells. In: 2014 International Conference on Advances in Computing, Communications and Informatics (ICACCI), pp 27932796 (2014).

[5] M. Matysek, M. Adamek, T. Karafiat (2016). The Mobile Monitoring and Control of Real Systems, in: Annals of DAAAM for 2016 \& Proceedings, DAAAM International, Viena, 2016, pp. 0104-0108.

[6] E.J. Hernandez-Valencia, M.C. Chuah (2000). Transport delays for umts voip. In: 2000 IEEE Wireless Communications and Networking Conference, vol 3, pp 1552-1556. WCNC (2000).

[7] G.J. Smith, G.W. Van Leeuwen (2004). Mobile communication optimization near wireless dead zone regions. US Patent 6,721,572 (2004).

[8] H. Kaaranen, et al. (2005). UMTS Networks: Architecture, Mobility and Services, John Wiley\&Sons, Oxford, 2005.

[9] M. Rumney (2009). LTE and the Evolution to 4G Wireless, John Wiley \& Sons, 2009.

[10] S. Fortes, A. Aguilar-García, R. Barco, F. Barba, J. Fernandez-luque, A. Fernández-Durán (2015). Management architecture for location-aware self-organizing lte/lte-a small cell networks. IEEE Commun. Mag. 53(1), 294-302, 2015.

[11] http://www.public.asu.edu/ jiayu2/geospark/ (2017). GeoSpark. A cluster computer framework for processing large-scale spatial data, Accessed on: 2017-10-21.

[12] http://www.qgis.org/en/site/about/index.html (2017). QGIS - The Leading Open Source Desktop GIS, Accessed on: 2017-10-21.

[13] https://hortonworks.com/about-us/ (2017). About Hortonworks, Accessed on: 2017-10-21. 